Z Rheumatol 2015 · 74:82-82

DOI 10.1007/s00393-014-1566-x

๑) Springer-Verlag Berlin Heidelberg 2015

Schriftleitung Zeitschrift für Rheumatologie

\title{
Die Zeitschrift für Rheumatologie dankt den Autoren der Rubrik CME - Zertifizierte Fortbildung 2014
}

\section{Prof. Dr. J. Grifka}

Orthopädische Klinik für die Universität Regensburg, Asklepios Klinikum Bad Abbach der Rubrik CME - Zertifizierte Fortbildung für ihre Unterstützung im Jahr 2014. Durch ihren Einsatz garantieren sie die hohe Qualität und klinische Relevanz der Rubrik in der Zeitschrift.

\section{Dr. S. Anders}

Orthopädische Klinik für die Universität Regensburg, Asklepios Klinikum Bad Abbach

\section{Prof. Dr. M. Aringer}

Medizinische Klinik und Poliklinik III, Abteilung Rheumatologie, Universitätsklinikum "Carl Gustav Carus" an der Technischen Universität Dresden

\section{Dr. X. Baraliakos}

Rheumazentrum Ruhrgebiet Herne

\section{PD Dr. Dr. S. Borgmann}

Klinische Infektiologie und Hygiene, Klinikum Ingolstadt

\section{Prof. Dr. J. Braun}

Rheumazentrum Ruhrgebiet Herne

\section{Prof. Dr. M. Fleck}

Klinik und Poliklinik für Rheumatologie/ klinische Immunologie, Asklepios Klinikum Bad Abbach

Klinik und Poliklinik für Innere Medizin I, Universitätsklinikum Regensburg

\section{Dr. M. Geyer}

Innere Medizin mit Schwerpunkt Rheumatologie, Justus-Liebig-Universität Gießen Abteilung für Rheumatologie und klinische Immunologie, Kerckhoff-Klinik, Bad Nauheim

\section{Prof. Dr. R. Gruber}

Institut für Labormedizin, Mikrobiologie und Krankenhaushygiene, Krankenhaus Barmher-

\section{Dr. N. Haustedt}

Klinik für Septische Knochen- und Weichteilchirurgie, Schön Klinikum Hamburg Eilbek, Hamburg

\section{Prof. Dr. G. Heers}

Orthopädische Klinik für die Universität Regensburg, Asklepios Klinikum Bad Abbach

\section{Dr. F. Heldmann}

Rheumazentrum Ruhrgebiet Herne

\section{Dr. W. Hermann}

Innere Medizin mit Schwerpunkt Rheumatologie, Justus-Liebig-Universität Gießen Abteilung für Rheumatologie und klinische Immunologie, Kerckhoff-Klinik, Bad Nauheim

\section{Dr. K. Holl-Ulrich}

Konsultations- und Referenzzentrum für Vaskulitisdiagnostik, Institut für Pathologie,Universitätsklinikum Schleswig-Holstein Campus Lübeck

\section{Dr. U. Kiltz}

Rheumazentrum Ruhrgebiet Herne

\section{Dr. J. Loock}

Klinik für Rheumatologie und klinische Immunologie, Schön Klinikum Hamburg Eilbek, Hamburg zige Brüder Regensburg

\section{Prof. Dr. F. Moosig}

Klinik für Rheumatologie und Immunologie, Universitätsklinikum SchleswigHolstein und Vaskulitisklinik, Klinikum Bad Bramstedt

\section{Dr. J.H. Schirmer}

Klinik für Rheumatologie und Immunologie, Universitätsklinikum SchleswigHolstein und Vaskulitisklinik, Klinikum Bad Bramstedt

\section{Dr. A.-K. Tausche}

Medizinische Klinik und Poliklinik III, Abteilung Rheumatologie, Universitätsklinikum "Carl Gustav Carus" an der Technischen Universität Dresden

\section{Prof. M. Vasile}

Abteilung für Innere Medizin und medizinische Spezialgebiete, Universität La Sapienza Rom

\section{S. Weigand}

Klinik und Poliklinik für Rheumatologie/ klinische Immunologie, Asklepios Klinikum Bad Abbach

\section{Prof. Dr. T. Witte}

Klinik für Immunologie und Rheumatologie, Medizinische Hochschule Hannover

Prof. Dr. J. Wollenhaupt

Klinik für Rheumatologie und klinische Immunologie, Schön Klinikum Hamburg Eilbek, Hamburg 